\title{
Reliability of the conditioned pain modulation paradigm to assess endogenous inhibitory pain pathways
}

\author{
Gwyn N Lewis $\mathrm{PhD}^{1}$, Luke Heales ${ }^{1}$, David A Rice BHSc ${ }^{1,2}$, Keith Rome $\mathrm{PhD}^{1}$, Peter J McNair PhD
}

\begin{abstract}
GN Lewis, L Heales, DA Rice, K Rome, PJ McNair. Reliability of the conditioned pain modulation paradigm to assess endogenous inhibitory pain pathways. Pain Res Manage 2012;17(2):98-102.
\end{abstract}

BACKGROUND: Conditioned pain modulation paradigms are often used to assess the diffuse noxious inhibitory control (DNIC) system. DNICs provide one of the main supraspinal pain inhibitory pathways and are impaired in several chronic pain populations. Only one previous study has examined the psychometric properties of the conditioned pain modulation technique and this study did not evaluate intersession reliability. OBJECTIVES: To evaluate and compare the intra- and intersession reliability of two conditioned pain modulation paradigms using different conditioning stimuli, and to determine the time course of conditioned pain inhibition following stimulus removal.

METHODS: An electronic pressure transducer was used to determine the pressure-pain threshold at the knee during painful conditioning of the opposite hand using the ischemic arm test and the cold pressor test. Assessments were completed twice on one day and repeated once approximately three days later.

RESULTS: The two conditioning stimuli resulted in a similar increase in the pressure-pain threshold at the knee, reflecting presumed activation of the DNIC system. Intrasession intraclass correlation coefficients for the cold pressor $(0.85)$ and ischemic arm tests $(0.75)$ were excellent. The intersession intraclass correlation coefficient for the cold pressor test was good $(0.66)$ but was poor for the ischemic arm test $(-0.4)$. Inhibition of the pressure-pain threshold remained significant at $10 \mathrm{~min}$ following conditioning, but returned to baseline by $15 \mathrm{~min}$.

CONCLUSIONS: Within-session reliability of DNIC assessment using conditioned pain modulation paradigms was excellent, but the applicability of assessing pain modulation over multiple sessions was influenced by the conditioning stimulus. The cold pressor test was the superior technique.

Key Words: Diffuse noxious inhibitory control; Pain; Reliability

$\mathrm{T}$ he conduction of nociceptive input from the spinal cord to supraspinal centres is strongly influenced by descending modulation from the brainstem. The diffuse noxious inhibitory control (DNIC) system provides one of the main descending pain inhibition pathways. The DNIC system, believed to be located in the caudal medulla (1), is activated when a painful stimulus is introduced to the body. The DNIC system focuses pain sensation on the stimulus by reducing concurrent pain input from remote sites of the body (2). Activation of the DNIC system is indicated by pain inhibition at anatomical regions of the body distant to where the new pain was introduced. The clinical importance of assessing the efficacy of the DNIC system is highlighted by the finding that DNIC-mediated inhibition is diminished in a range of chronic pain conditions (3-7). Moreover, in pain-free individuals, the magnitude of the DNIC response before surgery has been shown to predict which people will develop persistent pain after surgery (8). Thus, the measurement of DNIC function has widespread clinical relevance.

\author{
La fiabilité du paradigme de modulation \\ conditionnée de la douleur pour évaluer des voies \\ inhibitrices endogènes de la douleur
} HISTORIQUE : Les paradigmes de modulation conditionnée de la
douleur permettent souvent d'évaluer le système de contrôle inhibiteur dif-
fus nociceptif (CIDN). Le CIDN constitue l'une des principales voies
inhibitrices de la douleur sus-épineuse et est perturbé dans plusieurs popula-
tions souffrant de douleurs chroniques. Une seule étude a déjà porté sur les
propriétés psychométriques de la technique de modulation conditionnée de
la douleur, mais elle n'avait pas évalué la fiabilité entre les séances.
OBJECTIF : Évaluer et comparer la fiabilité pendant et entre les séances de
deux paradigmes de modulation conditionnée de la douleur au moyen de
divers stimulus de conditionnement, et déterminer l'évolution de l'inhibition
de la douleur conditionnée dans le temps après le retrait du stimulus.
MÉTHODOLOGIE : Un capteur de pression électronique a permis de déter-
miner le seuil de douleur du genou à la pression pendant un conditionnement
douloureux de la main opposée au moyen d'un test d'effort du bras sous
ischémie et d'une épreuve au froid. Les évaluations ont été effectuées deux fois
le même jour, puis répétées une fois environ trois jours plus tard.
RÉSULTATS : Les deux stimulus de conditionnement ont suscité une aug-
mentation similaire du seuil de douleur du genou à la pression, ce qui reflète
une activation présumée du système de CIDN. Les coefficients de corrélation
intraclasse étaient excellents au moyen de l'épreuve au froid ( 0,85$)$ et du test
d'effort du bras sous ischémie (0,75) au cours d'une même séance. Ce coeffi-
cient demeurait bon à l'épreuve au froid (0,66), mais était médiocre au test
d'effort du bras sous ischémie (-0,4) lorsqu'il était calculé sur plusieurs
séances. L'inhibition du seuil de douleur à la pression demeurait importante
dix minutes après le conditionnement, mais revenait à la normale au bout de
15 minutes.

CONCLUSIONS : L'évaluation du CIDN au moyen des paradigmes de modulation conditionnée de la douleur était d'une excellente fiabilité au cours d'une même séance, mais son applicabilité à de multiples séances était influencée par le stimulus de conditionnement. Lépreuve au froid était la technique qui fonctionnait le mieux.

The efficacy of the DNIC system is commonly measured in a laboratory setting by the conditioned pain modulation paradigm. This technique involves simultaneously applying two painful stimuli over distant regions of the body and assessing how much one (the conditioning stimulus) reduces the pain response evoked by the other (the test stimulus) (9). Because of the noxious requirement of the conditioning stimulus, it is presumed that the physiological mechanism underlying inhibition of the test stimulus involves the DNIC system. Conditioned pain modulation has been evaluated in this manner using a range of conditioning and test stimuli, including thermal (heat or cold), electrical, ischemic, mechanical and chemical stimuli (9). The inhibitory effects of conditioning are maximal during application of the conditioning stimulus and persist for several minutes after termination $(10,11)$, although the precise duration of inhibition is currently uncertain. Some reports have indicated that pain threshold returns to baseline within $5 \mathrm{~min}$ of stimulus cessation $(4,10-13)$ while others have reported effects lasting beyond 5 min poststimulus $(14,15)$. Some of the discrepancy between studies is likely to arise from the different

${ }^{1}$ Health and Rehabilitation Research Institute, AUT University; ${ }^{2}$ Pain Management Unit, Waitemata District Health Board,

Auckland, New Zealand

Correspondence: Dr Gwyn Lewis, Health and Rehabilitation Research Institute, AUT University, Private Bag 92006, Auckland 1142,

New Zealand. Telephone 64-9-921-9999, fax 64-9-921-9620, e-mail gwyn.lewis@aut.ac.nz 
conditioning and test stimuli that have been adopted, whether stimulus termination is defined as removal of the conditioning stimulus or when the conditioning pain has returned to zero, and the time interval that the assessments encompass. Indeed, it has been recently suggested that the procedures used to assess the efficacy of the DNIC system are standardized to enable easier comparison between studies (9).

The reliability of the conditioned pain modulation paradigm is also important to establish so that the significance of changes in DNIC efficacy over time or differences between populations can be evaluated. One previous study (16) investigated intrasession reliability of conditioned pain modulation and reported intraclass correlation coefficients (ICCs) that are considered fair-good according to standards indicated by Fleiss (17), although the associated CIs were not reported. In their study, Cathcart et al (16) used a manual pressure algometer to assess pressurepain threshold at the finger and shoulder during painful occlusion of the opposite arm. No study has currently reported the reliability of the conditioned pain modulation paradigm using an automated electronic pressure algometer, which may improve the consistency of threshold determination, or examined intersession reliability across days. The aims of the present study were to examine the intra- and intersession reliability of assessing DNIC function using a standardized assessment of pressure-pain threshold and the conditioned pain modulation paradigm; and to provide a detailed examination of the time course of conditioned pain inhibition after removal of the conditioning stimulus.

\section{METHODS}

\section{Participants}

Twenty healthy adults (mean $[ \pm \mathrm{SD}]$ age $25 \pm 8$ years, seven male) volunteered to participate in the study. Participants were excluded based on the following criteria: chronic pain in any part of the body or current pain in the lower limbs or back; a pathology affecting sensory perception; severe heart disease or respiratory conditions; current medication use (except for the oral contraceptive pill); or inability to provide informed consent and follow instructions of the study. The procedures conformed to the guidelines on pain research published by the International Association for the Study of Pain and were approved by the local ethics committee. Informed written consent was obtained from all participants.

\section{Pressure-pain threshold}

A custom-built, motor-driven electronic pressure transducer was used to assess pressure-pain threshold. Participants were positioned in long sitting on a height adjustable plinth with their right knee at $90^{\circ} \mathrm{flex}$ ion (Figure 1). The pressure probe had a diameter of $12 \mathrm{~mm}$ to activate primarily deep tissue nociceptors (18). It was positioned over the medial joint line of the right knee, $7.5 \mathrm{~cm}$ from the centre of the patella tendon. During threshold determination, the probe was pressed into the knee at a rate of $20 \mathrm{~N} / \mathrm{s}$. Participants were provided with a numerical pain scale from 0 to 100 with the anchors of $0=$ no sensation, 25 = discomfort, 50 = pain, 75 = intense pain, and $100=$ maximum tolerable pain (19). They were instructed to press a stop button immediately when they felt the stimulus had become just noticeably painful (a score of 50 on the pain scale). At each assessment of the pressure-pain threshold, two measurements were made approximately $30 \mathrm{~s}$ to $45 \mathrm{~s}$ apart, and the average of the two was determined as the threshold.

\section{Conditioning stimuli}

Two separate conditioning stimuli were used in the conditioned pain procedure. The order of conditioning stimuli was randomly assigned for the first session and remained the same for the second session. The ischemic arm test involved the placement of an inflatable cuff $(14.5 \mathrm{~cm})$ on the left arm just proximal to the cubital fossa. Participants completed $2 \mathrm{~min}$ of handgrip exercises before elevating their arm for $15 \mathrm{~s}$ (14). Immediately following this movement, the cuff was inflated to $240 \mathrm{mmHg}$ and the arm returned to the horizontal position for $2 \mathrm{~min}$. The pressure-pain threshold at the knee was assessed at $45 \mathrm{~s}$ and $90 \mathrm{~s}$ after cuff inflation.
The cold pressor test involved a water bath that was maintained at $12 \pm 1^{\circ} \mathrm{C}$. Participants immersed their left hand in the water bath up to the wrist crease for a period of $2 \mathrm{~min}$. The pressure-pain threshold at the knee was assessed at $45 \mathrm{~s}$ and $90 \mathrm{~s}$ after immersing the hand. During both conditioning stimuli, participants reported their subjective pain intensity on the numerical pain scale approximately $10 \mathrm{~s}$ after the second pressure-pain threshold assessment.

\section{Study protocol}

Participants attended two data collection sessions $3 \pm 0.5$ days apart (Figure 2). In the first session, pressure-pain threshold at the knee and conditioned pain modulation were assessed twice (measurement A and B) to evaluate intrasession reliability. Baseline knee pressure-pain threshold was first evaluated and then conditioned pain modulation was assessed by application of the first conditioning stimulus - either the cold pressor or ischemic arm test. After a 15 min break, the first conditioning stimulus was applied again and the pressure-pain threshold at the knee was reassessed. After an additional 15 min break, a second baseline pressure-pain threshold was established and the protocol repeated using the second conditioning stimulus.

At the second session, the same measures described above were assessed once (measurement $\mathrm{C}$ ) to establish the intersession reliability. Additionally, to examine the time course of conditioned pain inhibition, the pressure-pain threshold at the knee was obtained at 1, 5, 10 and 15 min following the termination of each conditioning stimulus $(n=19)$. To minimize circadian variations of pain regulation, testing was conducted at the same time of day for the two sessions. Participants were also asked to refrain from consuming caffeine, alcohol and nicotine and undertaking strenuous exercise for a period of $4 \mathrm{~h}$ before each session.

\section{Statistical analysis}

To evaluate the efficacy of the DNIC system, the difference in the pressure-pain threshold between baseline and noxious conditioning was determined, in which a positive change indicated an increase in threshold. The intrasession (measurement $\mathrm{A}$ and $\mathrm{B}$ ) and intersession (measurement A and C) reliability of knee pressure-pain threshold, conditioned pain inhibition and the numerical pain rating of each conditioning stimulus were evaluated using the ICC. ICC analyses were conducted using a two-way, mixed effects model with terms of absolute agreement (20). To examine the time course of inhibition, pressure-pain thresholds were analyzed using a one-way repeated measures ANOVA with the factor of time (baseline, during conditioning, 1, 5, 10, 15 min postconditioning). A Hunyh-Fedlt correction was used to adjust for the multiple comparisons. Paired $t$ tests were used to compare threshold values during and following conditioning to baseline if a significant ANOVA effect was found. The associated experimentwise error rate was also determined (21). The error rate indicated the percentage of results identified as statistically significant that were likely to be type I errors. All statistical analyses were conducted using SPSS version17 (IBM Corporation, USA). Data are presented as mean \pm SD.

\section{RESULTS}

Reliability of pressure-pain threshold

Across the three measurements, pressure-pain threshold at the knee averaged $47 \pm 14 \mathrm{~N}$. Table 1 shows the pressure-pain thresholds for measurements A, B and C and the intra- and intersession ICC reliability statistics. The intrasession ICC value indicated an excellent level of reliability while the intersession value was good (17).

\section{Reliability of assessing conditioned pain modulation}

Knee pressure-pain thresholds were substantially increased during the conditioning stimuli, reflecting an increase in pain tolerance at the right knee during painful stimulation of the left hand. Table 1 shows the difference in pressure-pain threshold at the three measurement points as well as the intra- and intersession ICC statistics. Across all 


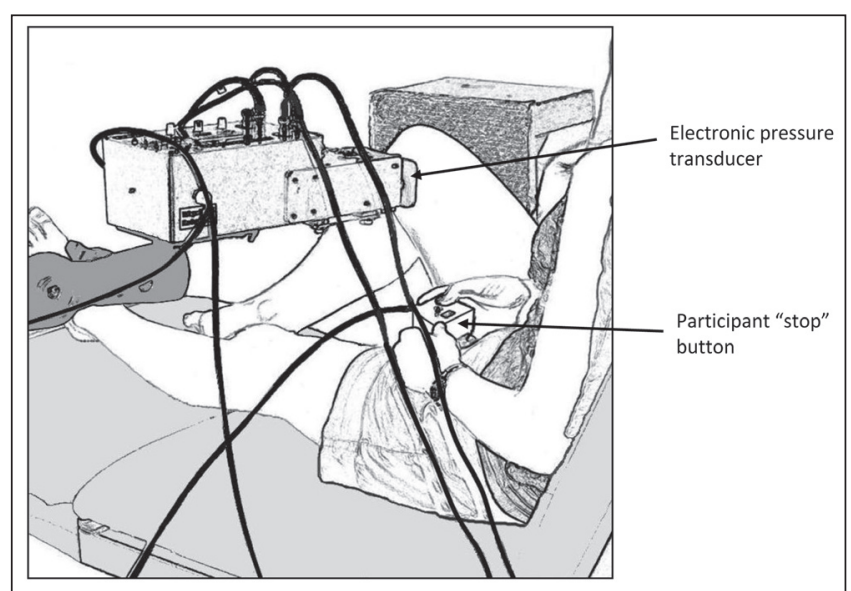

Figure 1) Diagram of the experimental set up. The participant was positioned in long-sitting with the right knee at $90^{\circ}$ flexion. A $12 \mathrm{~mm}$ diameter probe attached to the pressure transducer was positioned over the medial joint line of the knee. The probe exerted pressure onto the knee at a constant rate until the participant hit the 'stop' button, indicating the experience of pain

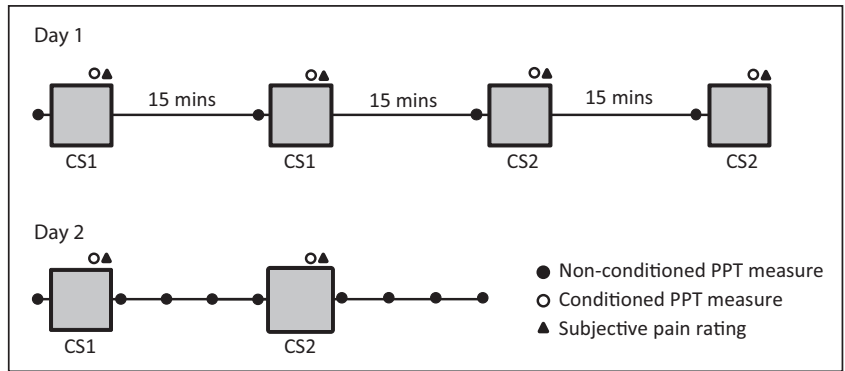

Figure 2) Schematic of the experimental protocol. On day 1, both conditioning stimuli were assessed twice, 15 min apart. On day 2, approximately three days later, the two conditioning stimuli were assessed once. The time course of conditioning was also evaluated on day 2 by assessing pressure pain threshold (PPT) at 1, 5, 10 and 15 min postconditioning. CS Conditioning stimulus

three measurement points, there was no difference in the change in pressure-pain threshold during the ischemic arm test compared with the cold pressor test $(\mathrm{P}=0.2)$. The intrasession ICC value for the change in pressure-pain threshold for the ischemic arm test suggested an excellent level of reliability but the intersession reliability was poor. The intrasession ICC value for the cold pressor test was again excellent and the intersession reliability was good.

Table 1 also shows the average pain scale rating for the two conditioning stimuli at each measurement point. Both conditioning stimuli produced a subjectively painful sensation with an average numerical pain scale score of $62 \pm 19$ and $73 \pm 18$ out of 100 for the ischemic arm and cold pressor tests, respectively. The lower bounds of the $95 \% \mathrm{CIs}$ of these pain ratings were above 50 for both conditioning stimuli, indicating that the conditioning stimuli were indeed painful. The cold

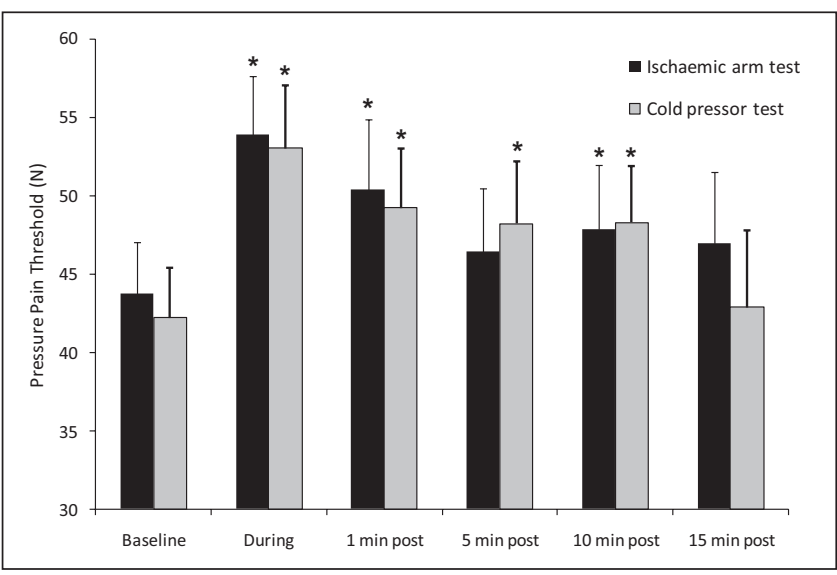

Figure 3) Group averages of pressure-pain threshold before, during and after application of the two conditioning stimuli. Error bars represent 1 SEM. * Statistically significant difference $(P<0.05)$ from baseline pressure-pain threshold

pressor test elicited significantly higher ratings on the pain scale compared with the ischemic arm test $(\mathrm{P}=0.003)$. The pain scale ratings for the ischemic arm test had fair-good intrasession and excellent intersession reliability. The pain scale ratings for the cold pressor test had excellent intra- and intersession reliability.

The relationship between numerical pain scale ratings and the extent of conditioned pain inhibition was also examined. Using the combined data from the three sessions for both conditioning stimuli, Pearson correlation analysis did not reveal any relationship between the subjective pain rating during conditioning and the change in pressure-pain threshold $(\mathrm{r}=0)$.

\section{Time course of conditioned pain inhibition}

Figure 3 shows the knee pressure-pain threshold during and following the two conditioning stimuli. The ANOVA revealed a significant effect of time for both the ischemic arm test $(\mathrm{F}[5,90]=4.6 ; \mathrm{P}=0.001)$ and the cold pressor test $(F[5,90]=3.3 ; \mathrm{P}=0.025)$. For the ischemic arm test the pressure-pain threshold was significantly higher than baseline during conditioning $(\mathrm{P}<0.001)$ and at $1 \mathrm{~min}(\mathrm{P}=0.015)$ and $10 \mathrm{~min}$ $(\mathrm{P}=0.026)$ following termination of the stimulus (experiment-wise error rate $=8 \%)$. There was no significant difference at $5 \mathrm{~min}(\mathrm{P}=0.2)$ and $15 \mathrm{~min}(\mathrm{P}=0.07)$. For the cold pressor test, the pressure-pain threshold was significantly higher than baseline during conditioning $(\mathrm{P}<0.001)$ and at $1 \mathrm{~min}(\mathrm{P}=0.002), 5 \mathrm{~min}(\mathrm{P}=0.027)$ and $10 \mathrm{~min}$ $(\mathrm{P}=0.023)$ following termination of the stimulus (experiment-wise error rate $=6 \%)$. There was no significant difference at $15 \mathrm{~min}$ $(\mathrm{P}=0.75)$.

\section{DISCUSSION}

The primary aim of the present study was to determine the reliability of using the conditioned pain modulation paradigm to assess DNIC function in a group of healthy participants. Establishing the reliability of a measure provides an indication of whether the measure is sufficient to identify differences between populations or in the same population over time. Overall, our conditioned pain modulation paradigm

TABLE 1

Measurement A, B and C, and intra- and intersession reliability data

\begin{tabular}{|c|c|c|c|c|c|}
\hline Measure & A & B & C & Intrasession ICC (95\% Cl) & Intersession ICC (95\% Cl) \\
\hline Knee PPT (N) & $51 \pm 15$ & $46 \pm 14$ & $42 \pm 12$ & $0.87(0.60-0.95)$ & $0.65(0.05-0.87)$ \\
\hline Ischemic arm test change (N) & $18 \pm 11$ & $12 \pm 13$ & $10 \pm 9$ & $0.75(0.35-0.90)$ & $-0.4(-1.8-0.4)$ \\
\hline Cold pressor test change $(\mathrm{N})$ & $12 \pm 16$ & $10 \pm 17$ & $11 \pm 10$ & $0.85(0.62-0.94)$ & $0.66(0.12-0.87)$ \\
\hline Ischemic arm test pain (NPS) & $65 \pm 21$ & $60 \pm 17$ & $63 \pm 21$ & $0.60(0.24-0.82)$ & $0.82(0.59-0.92)$ \\
\hline Cold pressor test pain (NPS) & $74 \pm 20$ & $71 \pm 19$ & $73 \pm 16$ & $0.94(0.86-0.98)$ & $0.80(0.56-0.92)$ \\
\hline
\end{tabular}

Data presented as mean \pm SD unless otherwise indicated. ICC Intraclass correlation coefficient; NPS Numerical pain scale; PPT Pressure-pain threshold 
exhibited excellent intrasession reliability; however, intersession reliability statistics were markedly different between the two conditioning stimuli, even though both gave rise to a similar change in the pressurepain threshold. Intersession reliability using the cold pressor test was fair-good, but the same statistic for the ischemic arm test was poor, questioning the ability of the latter conditioning stimulus to compare conditioned pain modulation across multiple testing sessions. For this reason, we recommend use of the cold pressor test in the future evaluation of DNIC pathways. The cold pressor test generates a more focal type of pain compared with the diffuse effects of ischemia, and this may contribute to its more consistent activation of descending inhibitory pathways.

Only one previous study has examined the intrasession reliability of the conditioned pain modulation paradigm and, to our knowledge, none has reported the intersession reliability. Our intrasession reliability measures were markedly higher than that reported by Cathcart et al (16). Measurement variability contributing to reliability estimates arises from two possible sources: the equipment and experimental procedures, and physiological variability within the population tested. Our use of a motor-driven electronic transducer may have reduced the variability of pressure-pain threshold measurements compared with assessment using a manual transducer. Although manual pressure-pain transducers have been shown to provide reliable estimates of pain threshold in previous studies (22-26), the more consistent rate of pressure application afforded by a motor driven system may still be beneficial. We also provided participants with a button switch to indicate pain threshold rather than use a spoken indication, which removes variability associated with the reaction time of the experimenter. A further difference in our conditioning stimuli is that we chose to use a standard protocol for conditioning rather than applying the conditioning stimulus until a specific numerical pain scale rating had been reached. We chose this method because previous studies have shown no relation between the subjective pain rating of the conditioning stimulus and the extent of inhibition elicited by the DNIC system (27-29). Overall, the cold pressor test elicited higher pain ratings, but there was no difference in the amount of inhibition elicited by the two conditioning stimuli. There was also no relationship between the subjective pain ratings and the extent of inhibition elicited by the two conditioning stimuli. Both of these findings support our justification for using a standard conditioning protocol.

A large extent of the variability in our measures, particularly that detected between sessions, is likely to arise from physiological variability within the nociceptive system. We attempted to minimize this variability by testing at a similar time of day for both sessions and restricting variables that are known to influence the pain modulatory system. One potential source of intersession variability in our study was the inclusion of female participants. The efficacy of the DNIC system is known to increase during the adulatory phase of the menstrual cycle (30). A possible limitation of our study is that this was not controlled in our female participants. However, given the brief duration of the adulatory phase, it is unlikely to have had a marked effect on our results. The small sample of male participants in our study $(n=7)$ also means that a separate analysis of these data would not be statistically appropriate. Another limitation was that we did not standardize the amount of handgrip exercise during the ischemic arm test, which may have contributed to the reduced reliability of this test. Given that the test still consistently elicited a painful response and the absence of a relationship between conditioning pain and the extent of pain inhibition, it is again unlikely to have a marked influence on our study findings.

Our analysis of the time course of conditioned pain inhibition showed that the effects of conditioning may persist for longer than previously believed. Pressure-pain threshold remained elevated at $10 \mathrm{~min}$ following both conditioning stimuli, but had returned to baseline by $15 \mathrm{~min}$. This finding suggests that multiple assessments of the nociceptive system within a session should be separated by a minimum of $15 \mathrm{~min}$ to ensure the lasting effects of conditioning have terminated.

\section{CONCLUSION}

The function of the DNIC system has been reported on in laboratory settings in both healthy and clinical populations. However, little work has addressed the reliability of its assessment. The present study showed that the assessment of DNIC function in healthy participants using a conditioned pain modulation procedure and a motor-driven electronic pressure transducer was reliable within a session; however, the reliability between sessions varied between our two conditioning stimuli. Although the two stimuli gave rise to a similar level of pain inhibition, the cold pressor test provided a more reliable conditioning stimulus that would be appropriate to use to assess DNIC function over multiple sessions. Further investigations of intra- and intersession reliability of the conditioned pain modulation paradigm should be conducted in clinical populations. We also recommended the adoption of a standardized conditioning protocol to improve the reliability and comparability of future investigations.

\section{REFERENCES}

1. Bouhassira D, Villanueva L, Bing Z, Le Bars D. Involvement of the subnucleus reticularis dorsalis in diffuse noxious inhibitory controls in the rat. Brain Res 1992;595:353-7.

2. Le Bars D. The whole body receptive field of dorsal horn multireceptive neurones. Brain Res Rev 2002;40:29-44.

3. Arendt-Nielsen L, Nie H, Laursen MB, et al. Sensitization in patients with painful knee osteoarthritis. Pain 2010;149:573-81.

4. Kosek E, Ordeberg G. Lack of pressure pain modulation by heterotopic noxious conditioning stimulation in patients with painful osteoarthritis before, but not following, surgical pain relief. Pain 2000;88:69-78

5. Lautenbacher S, Rollman GB. Possible deficiencies of pain modulation in fibromyalgia. Clin J Pain 1997;13:189-96.

6. Pielsticker A, Haag G, Zaudig M, Lautenbacher S. Impairment of pain inhibition in chronic tension-type headache. Pain 2005;118:215-23.

7. Sandrini G, Rossi P, Milanov I, Serrao M, Cecchini AP, Nappi G. Abnormal modulatory influence of diffuse noxious inhibitory controls in migraine and chronic tension-type headache patients. Cephalalgia 2006;26:782-9.

8. Yarnitsky D, Crispel Y, Eisenberg E, et al. Prediction of chronic post-operative pain: Pre-operative DNIC testing identifies patients at risk. Pain 2008;138:22-8.

9. Pud D, Granovsky Y, Yarnitsky D. The methodology of experimentally induced diffuse noxious inhibitory control (DNIC)-like effect in humans. Pain 2009;144:16-19.

10. Arendt-Nielsen L, Sluka KA, Nie HL. Experimental muscle pain impairs descending inhibition. Pain 2008;140:465-71.

11. Tuveson B, Leffler A-S, Hansson P. Time dependant differences in pain sensitivity during unilateral ischemic pain provocation in healthy volunteers. Eur J Pain 2006;10:225-32.

12. Campbell CM, France CR, Robinson ME, Logan HL, Geffken GR, Fillingim RB. Ethnic differences in diffuse noxious inhibitory controls. J Pain 2008;9:759-66.

13. Kakigi R. Diffuse noxious inhibitory control. Reappraisal by pain-related somatosensory evoked potentials following $\mathrm{CO}_{2}$ laser stimulation. J Neurol Sci 1994;125:198-205.

14. France CR, Suchowiecki S. A comparison of diffuse noxious inhibitory controls in men and women. Pain 1999;81:77-84.

15. Serrao M, Rossi P, Sandrini G, et al. Effects of diffuse noxious inhibitory controls on temporal summation of the RIII reflex in humans. Pain 2004;112:353-60.

16. Cathcart S, Winefield AH, Rolan P, Lushington K. Reliability of temporal summation and diffuse noxious inhibitory control. Pain Res Manag 2009;14:433-8.

17. Fleiss J. The design and analysis of clinical experiments. New York: John Wiley, 1986.

18. Treede RD, Rolke R, Andrews K, Magerl W. Pain elicited by blunt pressure: Neurobiological basis and clinical relevance. Pain 2002;98:235-40.

19. France CR, France JL, Absi M, Ring C, McIntyre D. Catastrophizing is related to pain ratings, but not nociceptive flexion reflex threshold. Pain 2002;99:459-63. 
20. Shrout P, Fleiss J. Intraclass correlations: Uses in assessing rater reliability. Psychol Bull 1979;86:420-8.

21. Ottenbacher KJ. Statistical conclusion validity. Multiple inferences in rehabilitation research. Am J Phys Med Rehabil 1991;70:317-22.

22. Potter L, McCarthy C, Oldham J. Algometer reliability in measuring pain pressure threshold over normal spinal muscles to allow quantification of anti-nociceptive treatment effects. Int J Osteopath Med 2006;9:113-9.

23. Chesterton LS, Sim J, Wright CC, Foster NE. Interrater reliability of algometry in measuring pressure pain thresholds in healthy humans, using multiple raters. Clin J Pain 2007;23:760-6.

24. Jones DH, Kilgour RD, Comtois AS. Test-retest reliability of pressure pain threshold measurements of the upper limb and torso in young healthy women. J Pain 2007;8:650-6.

25. Wylde V, Palmer S, Learmonth ID, Dieppe P. Test-retest reliability of quantitative sensory testing in knee osteoarthritis and healthy participants. Osteoarthritis Cartilage (In press).
26. Xiong S, Goonetilleke RS, Jiang Z. Pressure thresholds of the human foot: Measurement reliability and effects of stimulus characteristics Ergonomics 2011;54:282-93.

27. Granot M, Weissman-Fogel I, Crispel Y, et al. Determinants of endogenous analgesia magnitude in a diffuse noxious inhibitory control (DNIC) paradigm: Do conditioning stimulus painfulness, gender and personality variables matter? Pain 2008;136:142-9.

28. Lautenbacher S, Kunz M, Burkhardt S. The effects of DNIC-type inhibition on temporal summation compared to single pulse processing: Does sex matter? Pain 2008;140:429-35.

29. Nir R-R, Granovsky Y, Yarnitsky D, Sprecher E, Granot M. A psychophysical study of endogenous analgesia: The role of the conditioning pain in the induction and magnitude of conditioned pain modulation. Eur J Pain 2011;15:491-7.

30. Tousignant-Laflamme Y, Marchand S. Excitatory and inhibitory pain mechanisms during the menstrual cycle in healthy women. Pain 2009;146:47-55. 


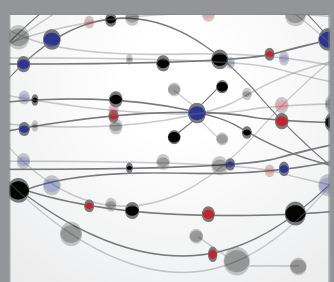

The Scientific World Journal
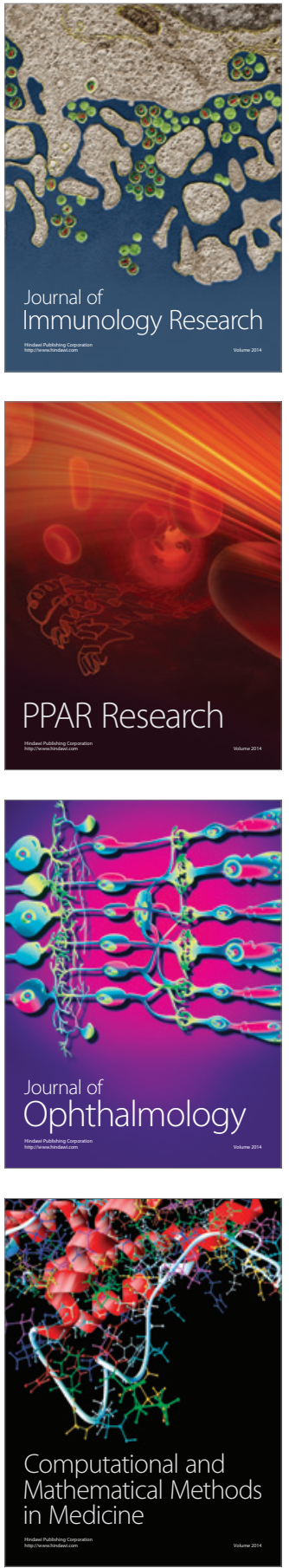

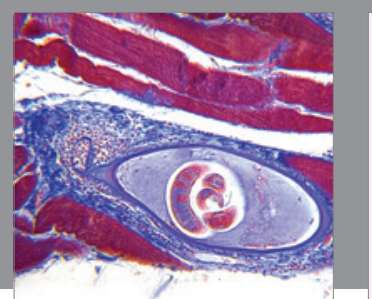

Gastroenterology Research and Practice

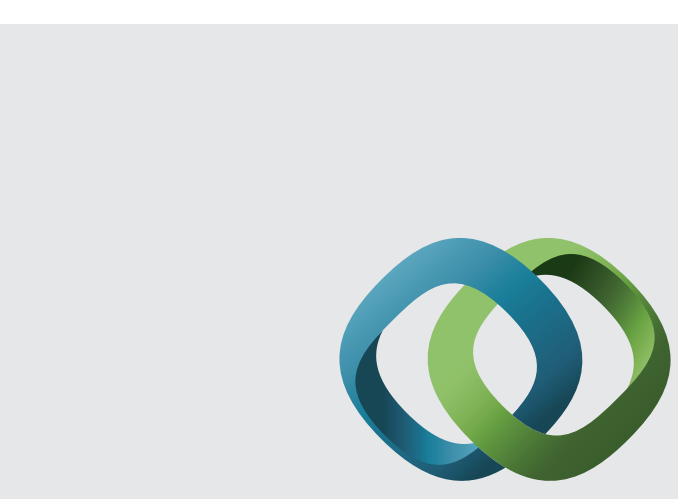

\section{Hindawi}

Submit your manuscripts at

http://www.hindawi.com
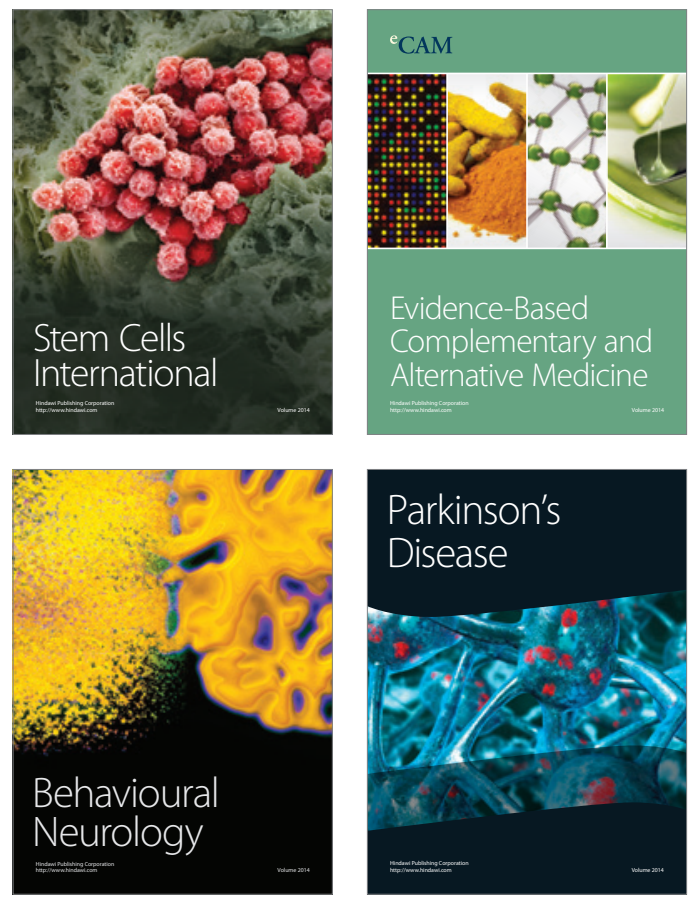
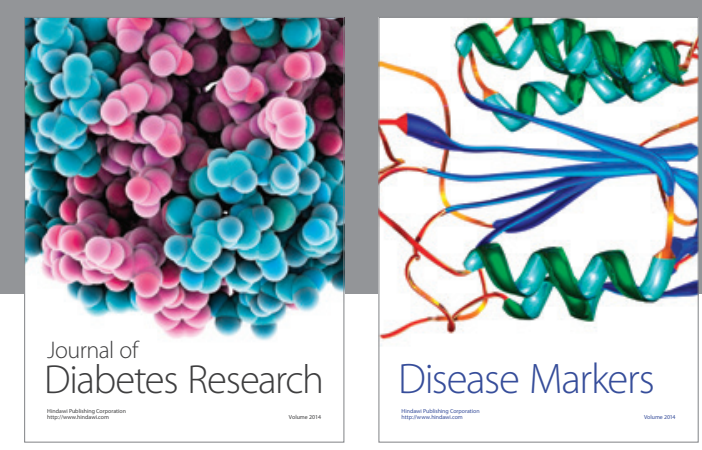

Disease Markers
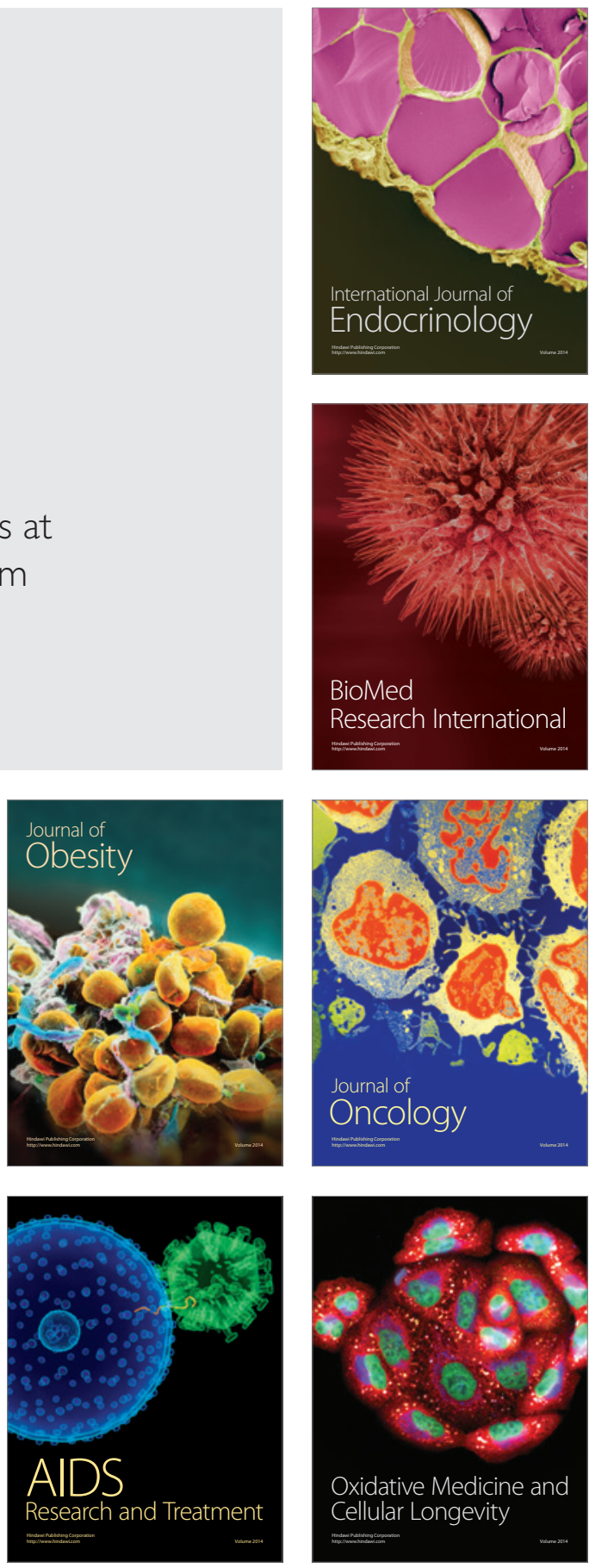